\title{
sciendo
}

Transport and Telecommunication, 2020, volume 21, no. 3, 191-202

Transport and Telecommunication Institute, Lomonosova 1, Riga, LV-1019, Latvia

DOI 10.2478/ttj-2020-0015

\section{REVIEW OF INVENTORY CONTROL MODELS: A CLASSIFICATION BASED ON METHODS OF OBTAINING OPTIMAL CONTROL PARAMETERS}

\author{
Ilya Jackson ${ }^{1}$, Jurijs Tolujevs ${ }^{2}$, Zhandos Kegenbekov $^{3}$ \\ ${ }^{1,2}$ Transport and Telecommunication Institute (TTI) \\ Lomonosova iela 1, Riga, Latvia \\ ${ }^{3}$ Kazakh-German University \\ Pushkin 111, Almaty, Kazakhstan \\ ${ }^{1}$ jackson.i@tsi.lv \\ ${ }^{2}$ tolujevs.j@tsi.lv \\ ${ }^{3}$ kegenbekov@dku.kz
}

\begin{abstract}
Inventory control has been a major point of discussion in industrial engineering and operations research for over 100 years. Various advanced numerical methods can be used for solving inventory control problems, which makes it a highly multidisciplinary filed attracting researchers from different academic disciplines. This fact makes it a daunting task to subsume the gargantuan spectrum of literature related to inventory control theory in one treatise. In light of this fact, this paper focuses on the timeline of inventory control models with respect to methodologies behind deriving optimal control parameters. Such methodologies include analytical approaches, optimal control theory, dynamic programming, simulation-based optimization and metamodel-based optimization.
\end{abstract}

Keywords: evolution of inventory control, classification of inventory models, optimal control parameters, review

\section{Introduction}

Mankind has been engaged in inventory control practices, at least in some primitive form, since ancient times, when it started to gather and stockpile resources of the planet. Moreover, inventory control has been a major point of discussion in industrial engineering and operations research for over 100 years (Bushuev et al., 2015). Various advanced numerical methods can be used for solving inventory control problems, which makes it a highly multidisciplinary filed attracting researchers from different academic disciplines. Despite the fact that the fundamental questions of inventory control theory are all about determining the proper timing and size of replenishment, each particular problem may be notably different from another. Furthermore, real-world problems are diverse and replete with specialties and subtleties. On the first hand, it emphasizes importance of inventory control models, and sparks the high degree of interest to study them. On the other hand, these facts make it a daunting task to subsume and summarize the gargantuan spectrum of literature related to inventory control theory in one treatise. In light of this fact, the paper focuses on timeline of inventory control models with respect to methodologies behind deriving optimal control parameters. Such methodologies include analytical approaches, optimal control theory, dynamic programming, simulation-based optimization and metamodel-based optimization.

Among other things, for comfortable further reading, it is crucially important to be familiar with the most common inventory policies that may be considered as an alphabet of inventory control. The models under consideration include such policies as:

- $(\mathrm{R}, \mathrm{S})$ policy assumes that the inventory level is reviewed every $\mathrm{R}$ period. Right after the review an order is placed bringing the inventory level to the predefined level $\mathrm{S}$;

- In $(\mathrm{R}, \mathrm{s}, \mathrm{S})$ policy the inventory level is also reviewed every $\mathrm{R}$ periods and as soon as it passes a reorder point $\mathrm{s}$, an order is placed bringing the inventory level to the predefined level $\mathrm{S}$;

- $(r, Q)$ policy is quite simple and straightforward, nevertheless, appears to be extremely efficient. According to this policy, the inventory level is reviewed continuously, and as soon as inventory level reaches the threshold $\mathrm{r}$, an order of size $\mathrm{Q}$ is placed;

- $(\mathrm{s}, \mathrm{S})$ policy is absolutely the same as $(\mathrm{R}, \mathrm{s}, \mathrm{S})$, besides the fact that the inventory level is reviewed continuously as in (r, Q) policy (Bartmann and Bach, 2012). 


\section{Methodology and structure}

This study has an overarching purpose to demonstrate the evolution of methods and practices in the field of inventory control theory in the form of a narrative review. On the other hand, the study does not aim to overview all the wide spectrum of literature related to inventory control theory. In this regard this study mentions scientific papers selectively in order to identify patterns and trends, characterize the state-of-the-art and guide future research.

The literature review has been performed using Scopus database and Google Scholar search engine focusing mainly on top tier and $2^{\text {nd }}$ tier journals and conference proceedings. At the preliminary phase such core keywords as "inventory control" and "inventory control model" were used. The collected literature was analyzed and it appeared that all the models and approaches can be conveniently classified by the method applied to derive optimal control parameters. After that the core keywords were combined with methodology-specific keywords that include "optimal control theory", "dynamic programming", "simulation-optimization" and "metamodel". The structured of the paper fully reflects the proposed classification (Figure 1).

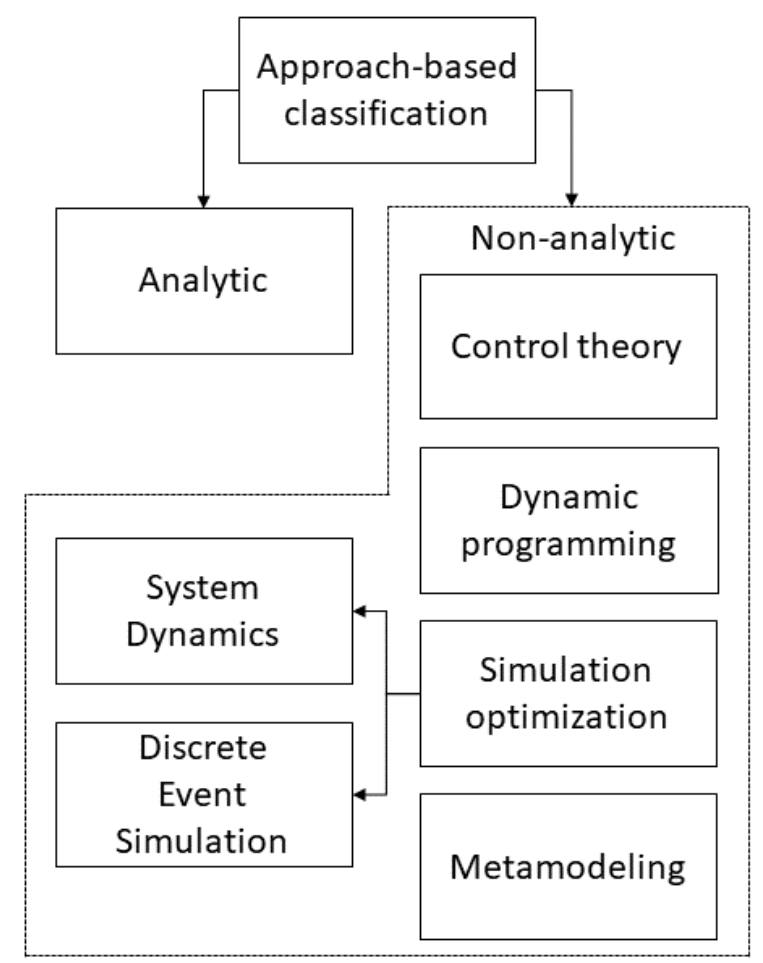

Figure 1. Approach-driven classification of inventory control models

\section{Analytic approach to inventory control}

Inventory control has occupied a steady niche among the most important topics in operations research. The history of mathematical theory of inventory and production may be traced back to Edgeworth (1888), who developed a variant of the news-vendor formula to model cash-flow in the bank. Nevertheless, the first formal model developed to aid managers in determining the proper size and timing of an inventory replenishment dates back to Harris (1913). The economic order quantity (EOQ) model is elementary, nevertheless, the most fundamental and celebrated:

$$
E O Q^{*}=\sqrt{2 \frac{K D}{h}}
$$

where $D$ is a constant demand rate (units produced per unit time), $K$ is a setup cost for a single unit and h is a holding cost charged per unit of time.

Because of its algebraic representation, it is well-known as "square-root-formula". The classical EOQ model makes such strict assumptions as prohibited backlogs and replenishment lead time equals to 
zero. Despite the simplicity, the equation elegantly describes the crucial relation in inventory control, namely the trade-off between replenishment and holding costs. This fact explains, why even nowadays several researchers still use EOQ model as a core complementing it with various extensions and superstructures (Lukinskiy and Lukinskiy, 2017). Important variations of this model include lost-sales, quantity discounts, shelf-life and non-zero replenishment lag.

EOQ-based models were followed by the study of models that incorporate both uncertainty and dynamics (Arrow et al., 1951). Models in which the demand flow is a random variable with a known probability distribution. This seminal paper was followed by the fundamental treatise in inventory theory (Arrow et al., 1958; Scarf, 1960). These works gave a rise to (R, s, S) and (s, S) policies, the most celebrated in inventory control theory. The $(\mathrm{R}, \mathrm{s}, \mathrm{S})$ policy was extensively studied, and its optimality under assumptions of independent and stationary demand and deterministic replenishment lead time was proved by Karlin (1960) for single-product inventory control systems. For stochastic single-product inventory control models, on the other hand, it was demonstrated that the (s, S) policy is optimal under different conditions. For instance, the optimality is proved for a case of fixed ordering and setup costs by Veinott (1966). Ten years later, optimality for the case of lost-sales was proven by Shreve (1976). However, as it was emphasized by Zipkin (2008), this proof does not extend to the case when the replenishment lead lag is non-zero.

The last-mentioned fact explicitly exposes the major drawback of analytic models, namely they put forward a set of assumptions and considerations that frequently do not correspond to real-world inventory control problems and do not guarantee the optimal solution, if these assumptions are violated. Taking into account that inventory control problems arise in various industries, and each single businessdriven problem is replete with non-standard factors and subtleties, it would be rash to believe that the same set of assumptions will be equally applicable to all inventory control systems. Besides such inflexibility, it is also recognized by such researchers as Duan and Liao (2013) and Tsai and Zheng (2013) that real-world inventory control problems are analytically intractable, due to their complexity and stochasticity. Such limitations of analytic approaches engendered new branches in studies of inventory control. Although, even nowadays classical analytic models are still developing and play an important role in inventory theory, a huge and diverse arsenal of non-analytic methods are applied, especially, in complicated business-driven problems.

\section{Control theoretical approach}

Currently optimal control theory is acknowledged as the completely developed branch of applied mathematics that uses differential equations to analyse, how physical systems behave in time (Ortega and Lin, 2004). Generally, differential equations are a classical way to model dynamics in the physical world. In this regard, its application to modelling of inventory control systems was considered a valid alternative. In the context of optimal control theory, a basic inventory dynamic can be expressed by the following differential equation:

$$
\frac{d I}{d t}=\operatorname{Poduction}(t-\tau)-\operatorname{Demand}(t),
$$

where the inventory dynamics $I(\mathrm{t})$ is the result of the difference between the inbound flow Production $(\mathrm{t}-\tau)$ and the demand rate Demand(t). Such that $\tau$ is a replenishment lag (Axsater, 1985).

Applications of classical optimal control methodology in inventory control can be traced back to Simon (1952), who managed to optimize inbound flow in an elementary single-product system. Sethi and Thompson (1981) attempted to apply stochastic differential equations to an industrial inventory control model. The considered model operated with a single product under stochastic and normally distributed demand. A straightforward application of optimal control theory for inventory systems was shown by Wikner (1994), who considered an industrial inventory-control model on three fundamental levels: forecasting, lead times, and inventory replenishment strategies. Laplace-transformations and Z-transformations were calculated for several common forecasting techniques including rolling averages and exponential smoothing.

Khmelnitsky and Gerchak (2002) paid specific attention on the fact that in some cases demand occasionally depends on the amount of available stock. This statement is especially true in cases of novelty or impulse purchases. The authors proposed optimal control theory-driven inventory model that incorporates dependence on inventory for demand rate and lost-sales. The paper provided an explicit solution for special case of time-invariant demand and incapacitated case is solved numerically and analysed in detail. The seminal study conducted by Benjaafar et al. (2010) considered the inventory 
control system that deals with "impatient customers". Researches managed to show that in such settings, optimal policy can be achieved using the modified (R, s, S). In a numerical study, the authors compared performance of the optimal policy against several other policies. More generalized model with probabilistic replenishment lags, allowed partial backordering and discounts was developed by Taleizadeh et al. (2017). This inventory control model was analysed scrupulously and a closed-form solution was obtained for the optimum order size.

Summing up, approaches based on control theory are, as a rule, proposed to a single-product inventory control system. As it was demonstrated such applications are both valid and efficient, when products are homogeneous enough to be treated as a flow.

\section{Dynamic programming approach}

Unfortunately, due to the "curse of dimensionality", at the scale of real-world business-driven problems both analytic and control theoretical models cannot be solved explicitly in reasonable computation time. In this regard, one must eventually resort to some sort of approximation (Sarimveis et al., 2008). On the other hand, as it was concluded by Domschke et al. (2015), inventory control problems can be naturally paraphrased as dynamic programs. The pivotal philosophy behind dynamic programming suggest linear separation of a problem into several sub-problems determining the solution functions recursively for each of them. Such that the combination of the partial problems' optima corresponds to the optimum of the original problem (Bellman, 1957).

Among numerous applications of dynamic programming, Clark and Scarf (1960) were the first to proof that the optimal feedback rule for a multi-product inventory control system is the (R, s, S) policy for the cases with static demand. The considered inventory control problem can be characterized as discretetime and finite-horizon. The optimal control parameters for the (R, s, S) policy were derived by splitting the original problem into a collection of single-echelon inventory control sub-problems with amended cost functions. Jammernegg (1981) meticulously studied a stochastic inventory control model with imperfect information on demand. Dynamic programming was applied to obtain optimal inventory control policies for two cases. Firstly, for a case of stochastic independent correlated demand. Secondly, for partially known and correlated demand. Dynamic programming was applied by Simchi-Levi and Zhao (2003) in order to analyse economic benefits from information sharing between a retailer and a producer in a multi-echelon supply chain. As it is mentioned by Cimen and Kirkbride (2017), as inventory control systems become more complex, approaches tailored for optimizing toy-like single-product systems are incapable of deriving optimal policies. For instance, optimal policies cannot be derived for inventory control systems involving the interrelated product inventories, multidimensionality and nondecomposability. In light of this fact, Cimen and Kirkbride proposed the application of approximate dynamic programming augmented with sample backup simulation approach to overcome such a computational challenge. The paper demonstrated numerical results that signify the undeniable advantage of policies developed with the proposed algorithm in comparison to policies obtained using deterministic approximation.

Summing up, dynamic programming is both popular and efficient procedure to obtain a nearlyoptimal policy for stochastic inventory control problems. However, dealing with large-scale multi-product multi-echelon inventory control systems, the computational burden becomes immense, which compels to incorporate approximation techniques along with simulation into the algorithmic framework.

\section{Simulation-based optimization}

Computer simulation or just simulation is the digital reproduction of a physical asset that relies on a computer to calculate the outcomes of a mathematical model in numerically straightforward manner. Since simulations allow one to verify the reliability of mathematical models in very flexible way, they have proven to be a useful tool for modelling of various physical systems including inventory (Dubois, 2018). Moreover, recent advances in simulation modelling and increased availability of computational capacity encouraged engineering, scientific and business communities to take advantage on simulations for modelling real-world problems for which the objective function cannot be expressed analytically (Pasupathy and Ghosh, 2013). As the result of increased popularity of simulations, natural interest in manipulating with input parameters in order to maximize or minimize the value of the objective function has sparked.

Simulation-optimization, also known as simulation-based optimization is an umbrella term for techniques that treat computer simulation as a "black-box" looking for specific settings of the input parameters that lead to the optimal output (Amaran et al., 2016). The real-world inventory control is 
commonly characterized by the necessity for nearly-optimal solutions in feasible computing times. That is why simulation-optimization techniques are used so widely to define an optimal inventory policy. Additionally, such an approach provides a modeler with a tool to deal with real-world stochasticity in unconstrained way and assess alternative candidate-solutions by risk analysis.

In general terms, in simulation-optimization problems an algorithm searches for such values of the decision variables that lead to the optimal output of the following objective function (Jalali and Nieuwenhuyse, 2015):

$$
\max _{x \in \Phi} J(x)=E[Y(x)]
$$

where $x$ stands for the vector of input parameters, and $\Phi$ is the set of feasible solutions. $Y(\mathrm{x})$ is a stochastic output of the simulation. So, the value of the objective function $J(\mathrm{x})$ is estimated based on the average of $n$ simulation runs (Koulouriotis et al., 2010).

$$
J_{n}(x)=\frac{1}{n} \sum_{i=1}^{n} Y(x)
$$

It is worth to emphasize that the number of runs (also known as replications) $n$ used in the estimation of objective function is a core determinant of both accuracy of estimation and computational cost for simulation-optimization techniques, which make it a subject of classical trade-off (Banks et al., 2000).

Summarizing, simulation-based optimization aims to utilize a simulation instead of an objective function in traditional form applying an optimization algorithm to find such simulation adjustments that would lead to the optimal output (Figure 2).

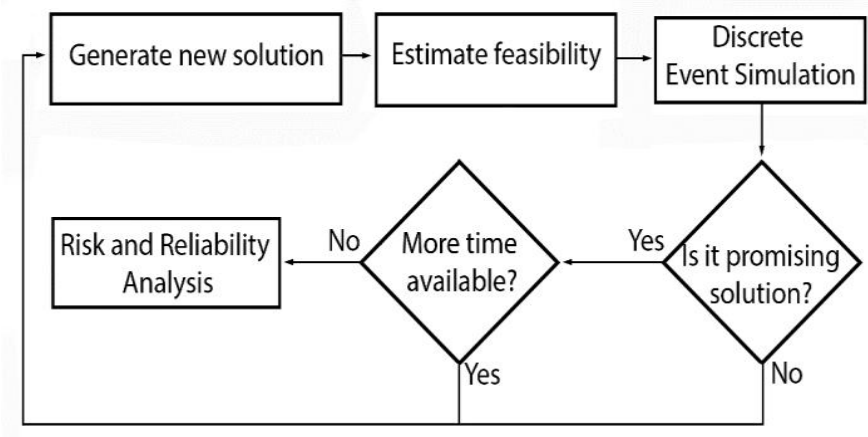

Figure 2. The logic behind simulation-optimization in discrete-event case

In the demonstrated framework, the iterative searching process has to assess the quality of feasible solutions, highlighting the promising ones. The process continues until the search time runs out. Immediately after this, a decision maker selects a final solution among promising with regard to a preferable risk policy. According to Pidd (1998), the simulation provides a natural way to introduce randomness of stochastic process. Furthermore, real-world stochasticity may be modelled throughout the best-fit probability distribution.

There is a plethora of simulation-optimization methods, however, all of them may be divided by two major groups, namely metaheuristics and gradient-based. When one deals with extremely large or even infinite set of feasible solutions, it is recommended to apply metaheuristics (Hong and Nelson, 2009). Notable examples of metaheuristics include genetic and evolutionary algorithms, evolutionary strategies, particle swarm optimization, tabu search and simulated annealing (Olafsson, 2006). Although metaheuristics is mainly applied to combinatorial optimization problems, they also could be successfully used in a continuous case (Andradottir and Prudius, 2010). For cases of continuous decision variables and differentiable objective function, such gradient-based methods as sample path optimization and stochastic approximation are widely used. It is worth to mention that such techniques require the gradient estimation, which may be done using likelihood ratio, finite differences, simultaneous perturbations, or perturbation analysis.

\subsection{System Dynamics}

In the late 1950s a novel methodology aimed to study dynamic flows in industrial systems was introduced (Forrester, 1961). This methodology was initially named as "Industrial Dynamics", however, the scope was greatly extended and the approach became eventually known as "System Dynamics" 
(Forrester, 1973). System Dynamics initially aimed to increasing the adoption of feed-back theory among managers and management scientists. At the beginning the method has been seriously criticized (Ansoff and Slevin, 1968). However, nowadays System Dynamics is a widely used method to understand the dynamics of complex systems including inventory control. The pivotal philosophy of the method is based on the holistic approach to the modelling, namely System Dynamics recognizes that the structure of any complex system is as important in determining its behaviour as the set of individual components themselves.

Despite the fact that System Dynamics is usually used exclusively as a simulation tool, the literature has several instances in which System Dynamics combined with optimization techniques was applied to identify optimal policies and parameters for inventory control systems (Schenk et al., 2010). For example, the paper by Wolstenholme (1982) presented the use of System Dynamics in optimization of multi-echelon supply chains in the copper industry. Besides inventory control, such aspects as mining and production were also taken into account. Additionally, it is worth to mention the case-study described by Powell and Bradford (2000), who managed to apply qualitative systems dynamics to derive the optimal resource-management policy (including inventory control parameters) for an international defence company.

\subsection{Discrete-Event Simulation}

Nowadays discrete-event simulation (DES) is the most prominent simulation paradigm for simulation-optimization frameworks (Gosavi, 2015). The DES paradigm is specialized in modelling systems at a low and medium level of abstraction and focuses on systems in which a sequence of operations is performed (Mahdavi and Wolfe-Adam, 2019). Following the introduction of linear congruential random-number generators (Lehmer, 1951), the origin of DES dates back to General Simulation Program, the first general-purpose simulator for industrial plant modelling (Tocher and Owen, 1960).

Nevertheless, the first simulation-based optimization of inventory control system was described only in the middle of $20^{\text {th }}$ century by $\mathrm{Fu}$ (1994), who demonstrated the simulation-optimization application to an inventory control system under $(\mathrm{s}, \mathrm{S})$ policy. The model assumed zero replenishment lag and periodic review. The cost function comprised holding, purchasing, transportation and backlogging. $\mathrm{Fu}$ managed to cover both the discrete parameter and the continuous parameter cases. In discrete parameter case, the author applied such methods as ranking-and-selection procedures and multiplecomparison. In the continuous parameter case such gradient-based methods as perturbation analysis were used. Applying derivative-free simulation-optimization method Kapuscinski and Tayur (1998) derived the nearly-optimal control policy and analysed its properties for a single-echelon inventory control model that operates with a single product under stochastic demand. Authors considered several cases including the finite-horizon and the discounted infinite-horizon. Even this early paper already concluded with an extremely significant statement that obtaining the exact analytical solutions is difficult for stochastic large-scale inventory control problems.

In the seminal paper Bollapragada and Rao (2006) examined a single-product, non-stationary inventory control problem with both supply and demand represented as random variables. Besides that, the model incorporated capacity limits on replenishment and service level requirements. A DES model for finite-horizon problem is developed in order to determine nearly-optimal replenishment orders over the horizon. The authors proposed an unusual heuristic based on the first two moments of the random variables and a normal approximation. Numerical experiments confirmed that the proposed heuristic was distinguished by high performance of approximately $99.75 \%$ of real optima. The applied simulationoptimization worked even when the output parameters were not normally distributed. Additionally, the paper contained sensitivity analyses of such parameters as shortage penalty costs, capacity limits, and demand variance. In the same year, the whole DES-based simulation-optimization framework was proposed (Aras et al., 2006). The framework was originally developed to compare two alternative strategies in realistic settings. The first strategy used manufacturing as the primary mean of satisfying customer demand. On the other hand, the second one was focused on remanufacturing. Simulationoptimization equipped with non-monotonic search heuristic was tested on numerical experiments with different initial parameters.

The solemn paper by Ding et al. (2009) addressed the design of production-distribution networks. Besides inventory control, such networks include supply chain configuration, order splitting and transport allocation. During the research a flexible framework for simulation was developed in order to enable the automatic simulation with different inventory control strategies. The framework incorporated a multi- 
objective genetic algorithm for optimization of operation strategies and associated control parameters in production-distribution network. It is also worth to note that the proposed simulation-optimization framework was applied in real-world automotive industry. The similar metaheuristics was utilized for optimization of stochastic multi-product inventory control system under the (R, s, S) policy (Arreola-Risa et al., 2011). Additionally, the proposed method incorporated regression analysis. The approach was developed and tested for an oil and gas company, which eventually decided to adopt it. In order to test the performance in general settings, 900 simulation experiment with different initial parameters were conducted.

Heuristics-driven simulation-optimization approach was also used for determination of the appropriate safety-stock level for inventory control in clinical trial supply chain (Chen et al., 2012). Besides inventory control, demand forecasting was a subject of interest. The heuristics behind the framework took advantage on mixed integer linear programming for solving the resource-constrained scheduling problem). Becerril-Arreola et al. (2013) considered a problem in online retail industry associated with two-step decision. In described settings, retailer firstly makes decisions on his profit margin and free-shipping threshold. After the optimal inventory level for a planning horizon should be determined. In the presented case-study publicly available statistics is used to find the best-fitting distribution for consumers" order sizes and conversion rate. The simulation software "Arena" was used to simulate the inventory control system and in-built optimizer "OptQuest" was applied to derive the optimal control parameters that lead to maximum profit. Moreover, a sensitivity analysis was conducted and important managerial insights were obtained. Namely, the impact of the unit holding and unit shipping costs on the retailer's optimal decisions was discovered. It is also worth to emphasize that some researches attempted to incorporate linear programming into simulation-optimization framework. For instance, Zeballos et al. (2013) in their unusual research embedded simplex method in order to find the optimal working capital target and order size for a single-product single-echelon finite horizon inventory control system. This study especially focused on financial aspects of inventory control incorporating into simulation such features as working capital constraints, transaction delays and several separated sources of financing.

Peirleitner, et al. (2016) considered a stochastic supply chain management problem. In this paper each node along the multi-echelon multi-product supply chain manages stock according to the (r, Q) policy. The problem was represented as bi-objective optimization problem. Such that overall supply chain costs were subject to minimization, while service level was subject to maximization. Such optimal control parameters as reorder points and lot sizes were found using simulation-optimization approach, which combined an evolutionary algorithm with DES simulation. In the same year discrete-rate simulation was used as a core to solve a single-product (r, Q) inventory control problem (Zvirgzdina and Tolujew, 2016). In this study the model was developed in ExtendSim using inbuilt genetic algorithm to derive optimal control parameters.

The research conducted by Zahedi-Hosseini (2018) is focused on the joint simulation-based optimization of operative maintenance and spare part inventory control for an industrial facility with various configurations. Firstly, spare part provision for a single-line conveyor-like system was considered. The simulation results indicate that a $(\mathrm{R}, \mathrm{s}, \mathrm{S})$ was cost-optimal. Secondly, a parallel multiline production system was modelled. It is found out that a policy inspired by just-in-time (r, Q) resulted the lowest costs. In both cases the long-run average cost per unit time was taken as an objective function to minimize. Several control policies were compared and optimal parameters were derived using the inbuilt optimizer. In order to tailor inventory control to urgent needs of grocery retail, the discrete-event simulation model with realistic perishability mechanics is proposed (Jackson, 2019a). The model is stochastic and operates with multiple products under constrained total inventory capacity. Additionally, the proposed model is distinguished by quantity discount, uncertain replenishment lags and lost sales. An optimisation framework based on a genetic algorithm is also proposed for deriving an optimal control policy.

\section{Metamodel-based optimization}

Unfortunately, simulation, especially detailed, is both time and memory consuming. Besides that, simulation-optimization algorithms do not "learn", more specifically, whenever the input parameters change slightly, a metaheuristic search (or another optimization method) must be executed once again. Taking into account a scale and dimensionality of real-world inventory control problems, such a neverending search becomes an unacceptable luxury for business. In the light of these facts, it is usually more reasonable to use an alternative cheaper-to-compute model, which is specifically designed in order to 
approximate an original simulation with a sufficient degree of accuracy. Such a "model of the model" is conventionally called a metamodel (Blanning, 1975).

Metamodeling is a fairly old and well-known approach in simulation community (Law and Kelton, 2000) that also has not bypassed logistics and production. The grist of the idea is to find a model that approximates the "black-box" with accuracy sufficient in the context of the task (Figure 3). As the result, a computationally efficient metamodel captures relations between decision variables and simulation output. After the metamodel is developed, it is possible to employ techniques specifically designed for deterministic optimization. Among various metamodeling techniques the most sought-after are response surface methodology (Angun et al., 2009), kriging models, radial basis functions (Biles et al., 2007) and artificial neural networks (Nezhad and Mahlooji, 2014).

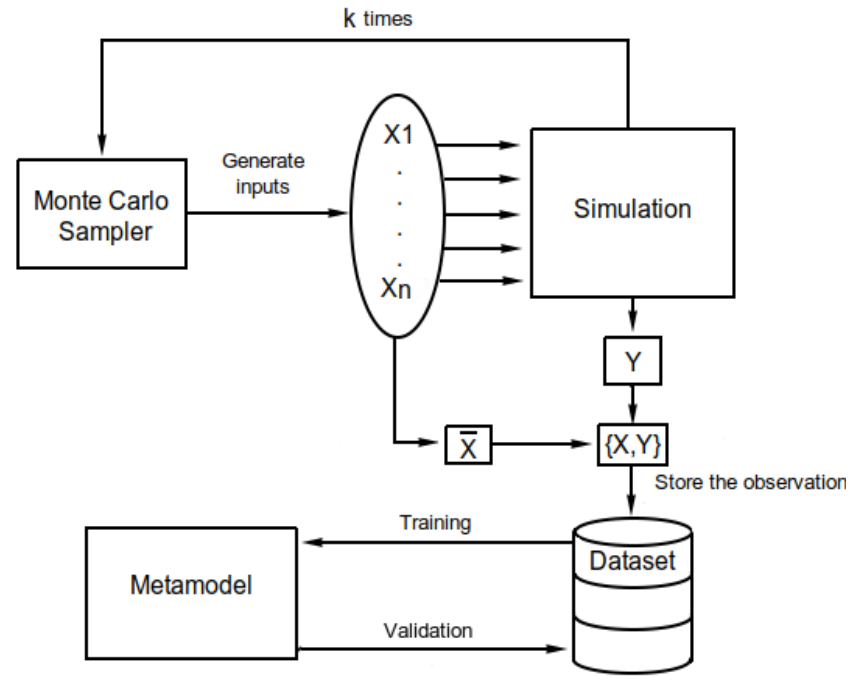

Figure 3. The logic behind metamodeling

Among applications related to inventory-control, it is, firstly, worth to mention the research conducted by Bendoly (2004). This research considered a simulation of substitute-inventory availability scenarios in transshipment inventory control system. The proposed methodology combined response surface methodology with non-linear optimization. The findings demonstrated that reductions in coordination costs and associated inventory availability decisions could provide limited benefits. Biles et al. (2007) explored the potential of the kriging methodology for constrained simulation optimization. The proposed technique is applied to an inventory control system under $(\mathrm{s}, \mathrm{S})$ policy. The experimental result demonstrated that kriging was a robust approach to solve constrained optimization problems in stochastic simulation of inventory control systems. Additionally, it is worth to mention the applied research (Lin et $a l ., 2009)$ that proposed an algorithm for defining an optimal inventory level for wafer fabrication processes. The research adopted a simulation model based on a real factory in order to generate data and demonstrates an optimization algorithm combining a multilayer feedforward neural network with quadratic programming. Combining second-order polynomial metamodel with response surface methodology the paper written by Angun (2011) demonstrated the solution to risk-averse SO problems. The novel robust response surface methodology was applied to inventory control problem with constrains.

Abruptly, recent pervasive and game-changing findings in deep-learning did not bypass metamodeling sparking the surge of interest to artificial neural networks (ANN) becoming a "hot-topic" in simulation community (Lechevalier et al., 2015). For example, Prestwich et al. (2012) managed to combine a single-layered ANN with an evolutionary algorithm to derive an optimal policy for a simulation-based stochastic multi-echelon inventory-control system. In the proposed neuroevolutionary approach ANN was trained by a simulation-driven evolutionary algorithm. Numerical experiments proved that this method is capable to derive high-quality policies in feasible computational time using networks of a simple architecture. In the same year the seminal research (Can and Heavey, 2012) presented another evidence of capability to combine genetic programming and ANNs for metamodeling of complex inventory control systems. This paper provided a comparative analysis of genetic programming and ANNs for metamodeling of DES models. Three stochastic industrial systems are empirically studied, namely an automated material handling system in semiconductor manufacturing, an 
$(\mathrm{s}, \mathrm{S})$ inventory control model and a serial production line. The results showed that genetic programming provides greater accuracy in validation tests, demonstrating a better generalization capability than ANN with one hidden layer. On the other hand, genetic programming required more computational budget comparing to metamodel-based approach.

Nezhad and Mahlooji (2014) meticulously studied ANN-based metamodels for stochastic multidimensional SO problems with constraints. It is important to emphasize that the authors used a realistic $(\mathrm{s}, \mathrm{S})$ inventory control model to study the robustness and efficiency of the developed algorithm and compared the results with those of the OptQuest optimization package. This numerical study indicated that the newfound metamodel-based algorithm was competitive in terms of accuracy, but required fewer simulation replications. It is also worth mentioning that Jad and Owayjan (2017) presented the simulation of a whole enterprise resource planning system that utilized 30-layered ANN as an inventory-policy controller. The application of neuroevolutionary automated machine learning to metamodeling of complex production-inventory systems with lost-sales and Markov-modulated demand is discussed in the recent research (Jackson, 2019b). The proposed solution incorporates multilayer perceptron and genetic algorithm.

\section{Conclusions}

For over a century inventory control has been a major point of discussion in academic, scientific and business worlds. Analytical models still hold the huge lay of the land in inventory control theory, especially in academia. Unfortunately, they do not guarantee the optimal solution, if the initial assumptions and considerations are violated. Moreover, such assumptions frequently do not correspond to real-world inventory control problems. On the other hand, real-world business-driven inventory control is frequently distinguished by multidimensionality, non-decomposability and replete with numerous specialties and subtleties.

Due to a high degree of complexity and analytically intractable of business-driven problems, inventory control theory adopts immense arsenal of methods from different scientific disciplines. For instance, the approaches based on control theory demonstrate efficiency in modelling of single-product inventory control systems, when products are homogeneous enough to be treated as a flow. On the other hand, the approaches based on dynamic programming are efficient in deriving nearly-optimal control parameters for stochastic inventory control problems. However, dealing with large-scale multi-product multi-echelon inventory control systems, the computational burden becomes immense, which compels to incorporate approximation techniques along with simulation into the algorithmic framework.

The tendency to choose computationally efficient numerical approximations over explicit analytic solutions rises the popularity of simulation-optimization techniques. A fortiori, simulation models may be exclusively tailored to industrial needs providing a modeler with a tool to deal with real-world uncertainty in unconstrained way. Unfortunately, simulation, especially detailed, is both time and memory consuming. In the light of these facts, it is commonly considered be more reasonable to use an alternative cheaper-to-compute metamodel, which is specifically built in order to approximate a target simulation with a sufficient degree of accuracy.

\section{References}

1. Amaran, S., Sahinidis, N., Sharda, B. and Bury, S. (2016) Simulation optimization: a review of algorithms and applications. Annals of Operations Research, 240(1), pp. 351-380.

2. Andradottir, S. and Prudius, A. (2010) Adaptive random search for continuous simulation optimization. Naval Research Logistics, 57, pp. 583-604.

3. Angun, E. (2011) A risk-averse approach to simulation optimization with multiple responses. Simulation Modeling Practice and Theory, 19, pp. 911-923.

4. Angun, E., Kleijnen, J., den Hertog, D. and Gurkan, G (2009) Response surface methodology with stochastic constraints for expensive simulation. Journal of the Operational Research Society, 60, pp. 735-746.

5. Ansoff, H. and Slevin, D. (1968) An appreciation of industrial dynamics. Management science, 14(7), pp. 383-397.

6. Aras, N., Verter, V. and Boyaci, T. (2006) Coordination and priority decisions in hybrid manufacturing/remanufacturing systems. Production and Operations Management, 15(4), pp. 528-543.

7. Arreola-Risa, A., Gimenez-Garcia, V. and Martinez-Parra, J. (2011) Optimizing stochastic production-inventory systems: A heuristic based on simulation and regression analysis. European Journal of Operational Research, 213(1), pp. 107-118. 
8. Arrow, K., Harris, T., and Marschak, J. (1951) Optimal Inventory Policy. Econometrica, 29, pp. 250-272.

9. Arrow, K., Karlin, S., and Scarf, H. (1958) Studies in the Mathematical Theory of Inventory and Production. Stanford, CA: Stanford University Press.

10. Axsater, S. (1985) Control theory concepts in production and inventory control. International Journal of Systems Science, 16, pp. 161-169.

11. Banks, J., Carson, J., Nelson, B., and Nicol, D. (2000) Discrete Event Systems Simulation. $3^{\text {rd }}$ edition. Prentice Hall, Englewood Cliffs, New Jersey.

12. Bartmann, D. and Bach, M. (2012) Inventory control: models and methods. Springer Science \& Business Media, p. 318.

13. Becerril-Arreola, R., Leng, M. and Parlar, M. (2013) Online retailers' promotional pricing, freeshipping threshold, and inventory decisions: A simulation-based analysis. European Journal of Operational Research, 230(2), pp. 272-283.

14. Bellman, R. (1957) Dynamic Programming. Princeton, Princeton University Press.

15. Bendoly, E. (2004) Integrated inventory pooling for firms servicing both on-line and store demand. Computers \& Operations Research, 31(9), pp. 1465-1480.

16. Benjaafar, S., Gayon, J. and Tepe, S. (2010) Optimal control of a production-inventory system with customer impatience. Operations Research Letters, 38(4), pp. 267-272.

17. Biles, W., Kleijnen, J., Van Beers, W. and Van Nieuwenhuyse, I. (2007) Kriging metamodeling in constrained simulation optimization: an explorative study. In: Proceedings of the 2007 Winter Simulation Conference. IEEE, pp. 355-362.

18. Blanning, R. (1975) The construction and implementation of metamodels, Simulation, 24, pp. 177-184.

19. Bollapragada, R. and Rao, U. (2006) Replenishment planning in discrete-time, capacitated, nonstationary, stochastic inventory systems. IIE Transactions, 38(7), pp. 605-617.

20. Bushuev, M., Guiffrida, A., Jaber, M. and Khan, M. (2015) A review of inventory lot sizing review papers. Management Research Review, 38(3), pp. 283-298.

21. Can, B. and Heavey, C. (2012) A comparison of genetic programming and artificial neural networks in metamodeling of discrete-event simulation models. Computers \& Operations Research, 39, pp. 424-436.

22. Chen, Y., Pekny, J. and Reklaitis, G. (2012) Integrated planning and optimization of clinical trial supply chain system with risk pooling. Industrial \& engineering chemistry research, 52(1), pp.152-165.

23. Cimen, M. and Kirkbride, C. (2017) Approximate dynamic programming algorithms for multidimensional flexible production-inventory problems. International Journal of Production Research, 55(7), pp. 2034-2050.

24. Clark, A and Scarf, H. (1960) Optimal policies for a multi-echelon inventory problem. Management Science, pp. 475-490.

25. Ding, H., Benyoucef, L. and Xie, X. (2009) Stochastic multi-objective production-distribution network design using simulation-based optimization. International Journal of Production Research, 47(2), pp. 479-505.

26. Domschke, W., Drexl, A., Klein, R. and Scholl, A. (2015) Einführung in Operations Research, 9, Berlin, Heidelberg: Springer Gabler.

27. Duan, Q. and Liao, T. (2013) Optimization of replenishment policies for decentralized and centralized capacitated supply chains under various demands. International Journal of Production Economics, pp. 194-204.

28. Dubois, G. (2018) Modeling and Simulation: Challenges and Best Practices for Industry. CRC Press.

29. Edgeworth, F. (1888) The Mathematical Theory of Banking. Journal of Royal Statistical Society, 51(1), pp. 113-127.

30. Forrester, J. (1961) Industrial Dynamics. Cambridge, MA, MIT Press, 36, pp. 37-66.

31. Forrester, J. (1973) World Dynamics. Cambridge, MA, MIT Press, pp. 17-29.

32. Fu, M. (1994) Sample path derivatives for (s, S) inventory systems. Operations Research, 42(2), pp. 351-364.

33. Gosavi, A. (2015) Simulation-based optimization. Berlin: Springer.

34. Harris, F. (1913) Operations and Cost. Factory Management Series. Chicago: A.W. Shaw and Company.

35. Hong, L. and Nelson, B. (2009) A brief introduction to optimization via simulation. In: Proceedings of the 2009 Winter Simulation Conference, pp. 314-322.

36. Jackson, I. (2019a) Simulation-Optimisation Approach to Stochastic Inventory Control with Perishability. Information Technology \& Management Science, 22, pp. 9-14. 
37. Jackson, I. (2019b) Neuroevolutionary Approach to Metamodeling of Production-Inventory Systems with Lost-Sales and Markovian Demand. In: Proceedings of the International Conference on Reliability and Statistics in Transportation and Communication. Springer, Cham, pp. 90-99.

38. Jad, F. and Owayjan, M. (2017) ERP Neural Network Inventory Control. Procedia Computer Science, 114, pp. 288-295.

39. Jalali, H. and Nieuwenhuyse, I. (2015) Simulation optimization in inventory replenishment: a classification. IIE Transactions, 47(11), pp. 1217-1235.

40. Jammernegg, W. (1981) On the optimality of generalized (s, t)-policies in production-inventory systems. Methods of Operations Research, 41, pp. 361-364.

41. Kapuscinski, R. and Tayur, S. (1998) A capacitated production-inventory model with periodic demand. Operations Research, 46(6), pp. 899-911.

42. Karlin, S. (1960) Dynamic Inventory Policy with Varying Stochastic Demands. Management Science, 6(3), pp. 231-258.

43. Khmelnitsky, E. and Gerchak, Y. (2002) Optimal control approach to production systems with inventory-level-dependent demand. Transactions on Automatic Control, 47(2), IEEE, pp. 289-292.

44. Koulouriotis, D., Xanthopoulos, A., and Tourassis, V. (2010) Simulation optimization of pull control policies for serial manufacturing lines and assembly manufacturing systems using genetic algorithms. International Journal of Production Research, 48, pp. 2887-2912.

45. Law, A. and Kelton, W. (2000) Simulation modeling and analysis. New York, McGraw-Hill.

46. Lechevalier, D., Hudak, S., Ak, R., Lee, Y. T., and Foufou, S (2015) A neural network meta-model and its application for manufacturing. In: Proceedings of the IEEE International Conference on Big Data, Santa Clara, USA.

47. Lehmer, D. (1951) Mathematical methods in large-scale computing units. Annual Computational Laboratory, 26, Harvard University, pp. 141-146.

48. Lin, Y., Shie, J. and Tsai, C. (2009) Using an artificial neural network prediction model to optimize work-in-process inventory level for wafer fabrication. Expert Systems with Applications, 36(2), pp. 3421-3427.

49. Lukinskiy, V. and Lukinskiy, V. (2017) Models of Inventory Management in Multi-level Distribution Systems. In: International Conference on Reliability and Statistics in Transportation and Communication, Springer, Cham, pp. 313-319.

50. Mahdavi, A. and Wolfe-Adam, T. (2019) Artificial Intelligence and Simulation in Business. White Paper, AnyLogic company, pp. 3-32.

51. Nezhad, A. and Mahlooji, H. (2014) An artificial neural network meta-model for constrained simulation optimization. Journal of the Operational Research Society, 65, pp. 1232-1244.

52. Olafsson, S. (2006) Metaheuristics. Handbooks in Operations Research and Management Science, 13, North Holland. Elsevier, pp. 633-654.

53. Pasupathy, R. and Ghosh, S. (2013) Simulation optimization: A concise overview and implementation guide. Operations Research, 7, INFORMS, pp. 122-150.

54. Peirleitner, A., Altendorfer, K. and Felberbauer, T. (2016) A simulation approach for multi-stage supply chain optimization to analyze real world transportation effects. In: Proceedings of the 2016 Winter Simulation Conference, IEEE, pp. 2272-2283.

55. Pidd, M. (1998) Computer simulation in management science. Chichester: Wiley.

56. Powell, J. and Bradford, J. (2000) Targeting intelligence gathering in a dynamic competitive environment. International Journal of Information Management, 20, pp. 181-195.

57. Prestwich, S. D., Tarim, S. A., Rossi, R. and Hnich, B. (2012) A neuroevolutionary approach to stochastic inventory control in multi-echelon systems. International Journal of Production Research, 50, pp. 2150-2160.

58. Sarimveis, H., Patrinos, P., Tarantilis, C. and Kiranoudis, C. (2008) Dynamic modeling and control of supply chain systems: A review. Computers \& operations research, 35(11), pp. 3530-3561.

59. Scarf, H. (1960) The Optimality of (s, S) Policies in the Dynamic Inventory Problem. Mathematical Methods in the Social Sciences. Stanford, CA: Stanford University Press, pp. 196-202.

60. Schenk, M., Tolujew, J. and Reggelin, T. (2010) A mesoscopic approach to the simulation of logistics systems. In: International Heinz Nixdorf Symposium. Springer, Berlin, Heidelberg, pp. 1525 .

61. Sethi, S. and Thompson, G. (1981) Dynamic optimal control models in advertising: recent developments. Management Science, 40, pp. 195-226.

62. Shreve, S. (1976) Abbreviated proof in the lost sales case. Dynamic Programming and Stochastic Control. New York: Academic Press, pp. 105-106. 
63. Simchi-Levi, D. and Zhao, Y. (2003) The value of information sharing in a two-stage supply chain with production capacity constraints. Naval Research Logistics, 50, pp. 888-916.

64. Simon, H. (1952) On the application of servomechanism theory in the study of production control. Econometrica, 20, pp. 247-268.

65. Taleizadeh, A., Zarei, H. and Sarker, B. (2017) An optimal control of inventory under probabilistic replenishment intervals and known price increase. European Journal of Operational Research, 257(3), pp. 777-791.

66. Tocher, K. and Owen, D. (1960) The automatic programming of simulations. In: Proceedings of the $2^{\text {nd }}$ International Conference on Operational Research. London. The English Universities Press Ltd.

67. Tsai, S. and Zheng, Y. (2013) A simulation optimization approach for a two-echelon inventory system with service level constraints. European Journal of Operational Research, 229, pp. 364-374.

68. Veinott, A. (1966) On the Optimality of (s, S) Inventory Policies: New Conditions and a New Proof. SIAM Journal on Applied Mathematics, 14(5), pp. 1067-1083.

69. Wikner, J. (1994) Dynamic modelling and analysis of information flows in production-inventory and supply chain systems. Profil, Linkoping.

70. Wolstenholme, E. (1982) Managing international mining - case study concerning complex ownership systems. European Journal of Operational Research, 9, pp. 133-143.

71. Zahedi-Hosseini, F. (2018) Modeling and simulation for the joint maintenance-inventory optimization of production systems. In: Proceedings of the 2018 Winter Simulation Conference, IEEE Press, pp. 3264-3274.

72. Zeballos, A., Seifert, R. and Protopappa-Sieke, M. (2013) Single product, finite horizon, periodic review inventory model with working capital requirements and short-term debt. Computers \& Operations Research, 40(12), pp. 2940-2949.

73. Zipkin, P. (2008) Old and New Methods for Lost-Sales Inventory Systems. Operations Research, 56(5), pp. 1256-1263.

74. Zvirgzdina, B. and Tolujew, J. (2016) Experience in Optimization of Discrete Rate Models Using ExtendSim Optimizer. In: Proceedings of the $9^{\text {th }}$ International Doctoral Students Workshop on Logistics, June, 2016.Magdeburg, Otto von Guericke University, pp. 95-101. 